\title{
Characterization of magnetic biochar amended with silicon dioxide prepared at high temperature calcination
}

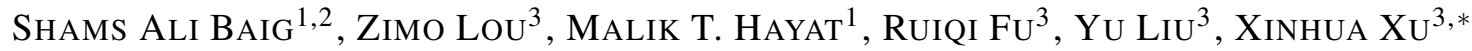 \\ ${ }^{1}$ Department of Environmental Sciences, COMSATS Institute of Information Technology (CIIT), Abbottabad 22060, Pakistan \\ ${ }^{2}$ Department of Environmental Sciences, Abdul Wali Khan University, Mardan 23200, Pakistan \\ ${ }^{3}$ Department of Environmental Engineering, College of Environmental and Resource Sciences, Zhejiang University, \\ Hangzhou 310058, People's Republic of China
}

\begin{abstract}
Calcination is considered to increase the hardness of composite material and prevent its breakage for the effective applications in environmental remediation. In this study, magnetic biochar amended with silicon dioxide was calcined at high temperature under nitrogen environment and characterized using various techniques. X-ray diffraction (XRD) analysis revealed elimination of $\mathrm{Fe}_{3} \mathrm{O}_{4}$ peaks under nitrogen calcination and formation of $\mathrm{Fe}_{3} \mathrm{Si}$ and iron as major constituents of magnetic biochar- $\mathrm{SiO}_{2}$ composite, which demonstrated its superparamagnetic behavior $\left(>80 \mathrm{Am}^{2} \cdot \mathrm{kg}^{-1}\right)$ comparable to magnetic biochar. Thermogravimetric analysis (TGA) revealed that both calcined samples generated higher residual mass $(>96 \%)$ and demonstrated better thermal stability. The presence of various bands in Fourier transform infrared spectroscopy (FT-IR) was more obvious and the elimination of $\mathrm{H}-\mathrm{O}-\mathrm{H}$ bonding was observed at high temperature calcination. In addition, scanning electron microscopy (SEM) images revealed certain morphological variation among the samples and the presence of more prominent internal and external pores, which then judged the surface area and pore volume of samples. Findings from this study suggests that the selective calcination process could cause useful changes in the material composites and can be effectively employed in environmental remediation measures.
\end{abstract}

Keywords: calcination; magnetic biochar; material composite; saturation magnetization

(C) Wroclaw University of Technology.

\section{Introduction}

Calcination process is considered to increase hardness of different composite materials and prevent their breakage [1]. Generally, high temperature selective environment-based calcination alters material textural and mineralogical characteristics, which for some composite materials improves surface properties and attracts their environmental applications [2]. However, Mahmood et al. [3] found reduction of pollutants removal in mixed oxides calcined at higher temperature. Numerous powdered materials have been utilized for toxic metals removal from aqueous systems, but were not suitable to employ in column-based treatments [4].

Recent studies revealed that magnetic biochar works as multifunctional material and is widely

*E-mail: xuxinhua@zju.edu.cn employed for the bioremediation of various toxic pollutants from soil and water environments [5-7]. However, the powdered magnetic biochar cannot be detached from aqueous treatment systems and also cannot be used in column-based treatments [8, 9]. Various granulation processes may help to produce required size granules for targeted environmental applications [9]. Granulation is associated with the cutting or shredding a large object into small granules or compressing of fine powders to create bonds between them to form granules [10]. For instance, weak gel was applied as a binding agent to make red mud granules [11]. In our previous study [12], the composite material containing $\mathrm{Fe}_{3} \mathrm{O}_{4}$ and $\mathrm{SiO}_{2}$ as the major constituents unexpectedly transformed into a new phase with larger granules during high temperature calcination under nitrogen environment. In contrast, high temperature calcination under dynamic air can assist 
to form segregated magnetite, hematite and quartz from the same composite material. In addition, silica coating was reported to effectively protect iron oxide particles from aggregation and sintering at higher temperature [13]. Similarly, coal ash was granulated using $10 \%$ to $15 \%$ cement as a binding agent in granulation process and was used commercially for pollution control measures [14]. Therefore, we hypothesized that the application of calcination for magnetic biochar amended with $\mathrm{SiO}_{2}$ may change the morphological and mineralogical properties of the composite material and increase the granules size, which further can be used in pollution control measures. In addition, the effect of calcination on textural, mineralogical, and magnetic properties of magnetic biochar amended with $\mathrm{SiO}_{2}$ has seldom been studied in literature.

In this study, magnetic kans grass biochar was amended with $\mathrm{SiO}_{2}$ and calcined at $1000{ }^{\circ} \mathrm{C}$ under nitrogen environment to investigate the composite material textural, mineralogical, and magnetic variations using different characterization techniques (i.e. XRD, FT-IR, SEM, EDX, TGA, BET and saturation magnetization). Five samples: kans grass biochar (KGB), magnetic kans grass biochar (MB), magnetic biochar-calcined (MBC), magnetic biochar amended with $\mathrm{SiO}_{2}$ (MBS) and magnetic biochar amended with $\mathrm{SiO}_{2}$-calcined (MBSC) were synthesized and investigated. This study let us assume that it would be a further explored new research direction to prepare surface effective composite materials from powdered material (i.e. biochar) using different granulation techniques (e.g. calcination).

\section{Materials and methods}

\subsection{Preparation of composite materials}

Kans grass (Saccharum spontaneum) was collected from a wetland in Hangzhou city of China, as described earlier [7]. The sample was placed in a tightly covered stainless steel trays and pyrolyzed in a muffle furnace $\left(\mathrm{SX}_{2}\right.$ Shanghai Rong Feng Scientific Instrument, Inc.) under oxygen limited environment. The pyrolysis temperature was raised at a heating rate of $26{ }^{\circ} \mathrm{C} \mathrm{min}-1$ until it reached
$500{ }^{\circ} \mathrm{C}$ and held for $4 \mathrm{~h}$. After cooling, the biochar was taken out and meshed $(\sim 0.154 \mathrm{~mm})$ for further applications. The dried kans grass biochar (KGB) was fabricated with $\mathrm{Fe}^{3+} / \mathrm{Fe}^{2+}$ by chemical coprecipitation [15] to obtain biochar $/ \mathrm{Fe}_{3} \mathrm{O}_{4}$ composite, which has been referred as magnetic biochar (MB). To prepare magnetic biochar-SiO $\mathrm{SiO}_{2}$ (MBS) composite, supernatants from MB aliquot were removed and appropriate amounts of ethanol, ammonia and DI were successively added. Then, the solution was sonicated for $1 \mathrm{~h}$. In addition, a calculated amount of TEOS (tatraethoxysilane) was added and the reaction was held for $2 \mathrm{~h}$. The solution was kept at $60{ }^{\circ} \mathrm{C}$ for overnight after the addition of $50 \mathrm{~mL}$ ethanol. Afterward, the aliquot was washed using de-oxygenated DI water for several times to remove all the impurities. MBS materials were separated from the solution using external magnetic field and dried under vacuum at $60{ }^{\circ} \mathrm{C}$ for $24 \mathrm{~h}$. The dried MBS was further processed by calcination to prepare a calcined product. Briefly, a proportional amount of MBS was added into the muffle furnace at $1000{ }^{\circ} \mathrm{C}$ under nitrogen atmosphere for $2 \mathrm{~h}$ to obtain another product, referred as magnetic biochar amended with $\mathrm{SiO}_{2}$-calcined (MBSC).

\subsection{Sample characterizations}

Fourier transform infrared spectroscopy (FT-IR) (IR Prestige-21, Japan) was performed using $\mathrm{KBr}$ disc method and measured as wavenumber $\left(\mathrm{cm}^{-1}\right)$. X-ray diffraction patterns (XRD) of the samples were recorded on X'Pert PRO analytical B.V., Netherlands, at 2-theta range $\left(10^{\circ}\right.$ to $90^{\circ}$ ). Scanning electron micrograph (SEM) (obtained with Hitachi S-3000N, Japan) was used to investigate the morphological compositions of the samples. Surface area was determined using BET surface analyzer (ASAP 2050, Micrometrics, Beijing, China). Quantum design PPMS-9 magnetometer was used to measure the magnetic properties in the magnetic field ranging from -20000 Oe to 20000 Oe at room temperature. X-ray energy dispersive spectrometer (EDS, model: ESM-5800, GEOL, Japan) was used to measure the elemental compositions. 


\section{Results and discussion}

\subsection{XRD patterns}

Fig. 1 presents the XRD patterns of the prepared KGB, MB, MBC, MBS and MBSC samples. The diffraction peak indexed as $\left(\begin{array}{lll}0 & 0 & 2\end{array}\right)$ in the KGB sample could be assigned the noncrystalline hemicellulose [16], which still remained after the pyrolysis of the kans grass. Magnetite and quartz were found to be the major constituents in MB and MBS samples, as revealed in Fig. $1 \mathrm{~b}$ and 1d. Magnetite diffraction peaks appeared at $2 \theta=30.0^{\circ}, 35.3^{\circ}$, $56.8^{\circ}$, and $62.3^{\circ}$, which were assigned to $(220)$, (3 11 1), (5 111 ) and (4 $\left.4 \begin{array}{ll}4 & 0\end{array}\right)$ planes, respectively. However, after high temperature calcination under nitrogen most of the magnetite peaks were either reduced or converted into other constituents, which is in agreement with the findings of our previous studies [12, 17]. However, in this study, the compositions of the new phases were different, as compared to the findings from our earlier studies and the reason could be the differences in precursor material compositions. The major peaks appeared at $2 \theta=44.7^{\circ}, 64.9^{\circ}, 82.4^{\circ}$ which could be assigned to (1 110$),\left(\begin{array}{lll}2 & 0 & 0\end{array}\right)$ and ( $\left.\begin{array}{lll}2 & 1 & 1\end{array}\right)$ planes iron $(\mathrm{Fe})$, as shown in Fig. 1e. In addition, few major diffraction peaks at $2 \theta=45.2^{\circ}, 66.0^{\circ}$ and $87.7^{\circ}$ in MBSC samples could be assigned to $\mathrm{Fe}_{3} \mathrm{Si}$, which was formed at high temperature calcination under nitrogen. An increase in elemental compositions, particularly of silicon and iron contents was observed in EDX analysis, as given in Table 1. But the oxygen content decreased drastically in the calcined samples (Table 1). Furthermore, the quartz

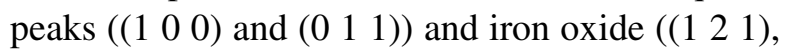
$\left(\begin{array}{lll}-1 & 1 & 0\end{array}\right)$ and $\left.\left(\begin{array}{lll}1 & 2 & 0\end{array}\right)\right)$ with the assigned planes, respectively also disappeared in the samples calcined under nitrogen environment.

\subsection{FT-IR analyses}

Fig. 2 presents the FT-IR spectra of KGB, MB, MBC, MBS and MBSC samples. The presence of various bands in FT-IR spectra indicates various functional groups present in the composite samples [5]. The band appearing at $475 \mathrm{~cm}^{-1}$ could be attributed to $\mathrm{Fe}-\mathrm{O}$ vibration, which was found to

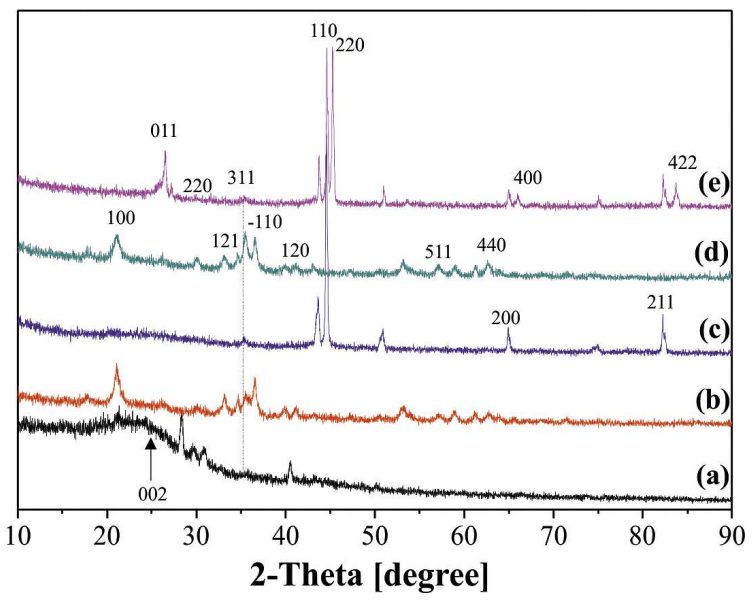

Fig. 1. XRD patterns of (a) KGB, (b) MB, (c) MBC, (d) MBS and (e) MBSC.

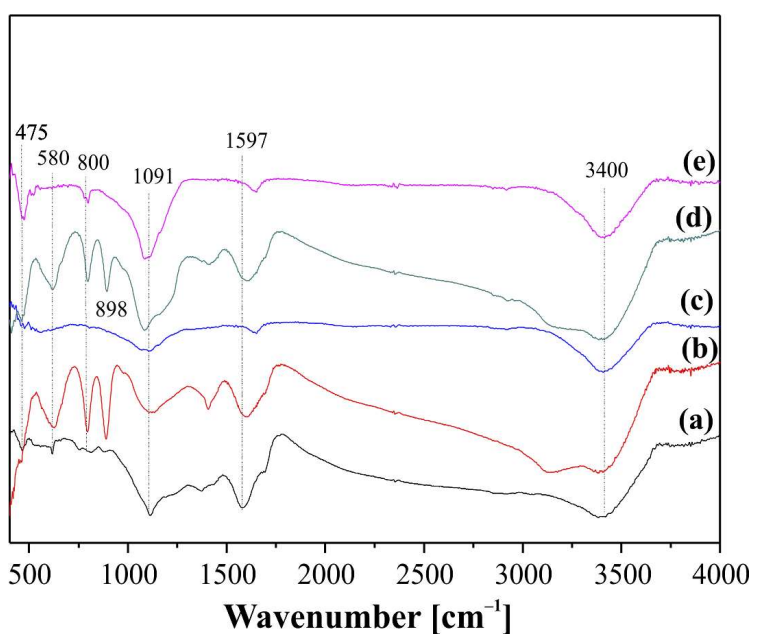

Fig. 2. FT-IR spectra of (a) KGB, (b) MB, (c) MBC, (d) MBS and (e) MBSC.

become stronger in the sample calcined at higher temperature (Fig. 2e). Visible bands appearing around $580 \mathrm{~cm}^{-1}, 800 \mathrm{~cm}^{-1}$ and $898 \mathrm{~cm}^{-1}$ correspond to the available organic residues, such as aromatic $\mathrm{C}-\mathrm{C}$ bonding in lignin, polysaccharide and polymeric $\mathrm{CH}_{2}$, which were found to be eliminated in both the samples (MBC and MBSC) after calcination at higher temperature. Similarly, higher temperature calcination eliminated $\mathrm{H}-\mathrm{O}-\mathrm{H}$ bonding at around $1550 \mathrm{~cm}^{-1}$ to $1597 \mathrm{~cm}^{-1}$ in water, as shown in Fig. $2 \mathrm{c}$ and Fig. 2e, but in other samples it was assumed to be responsible for a wider band [17]. 


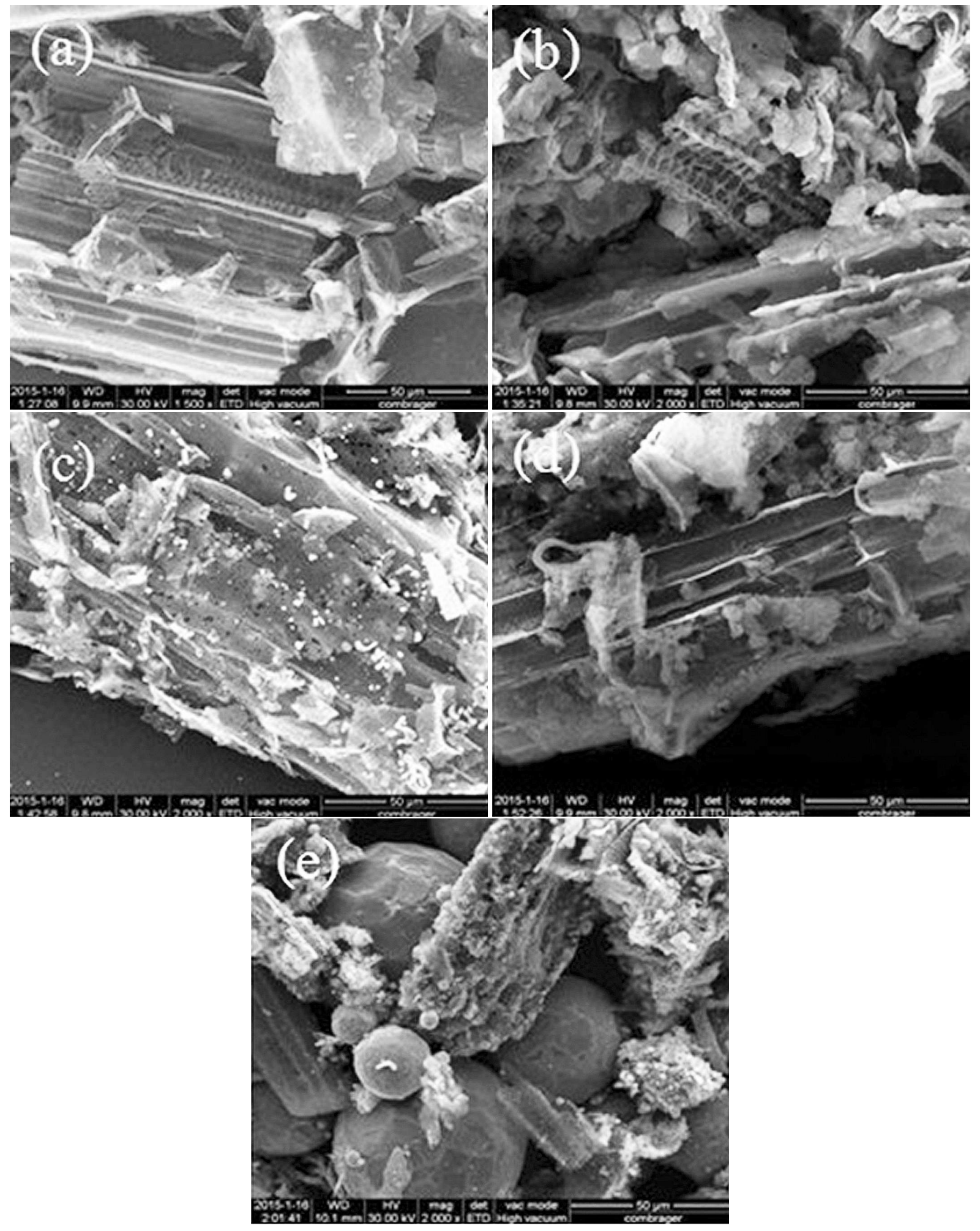

Fig. 3. SEM images of (a) KGB, (b) MB, (c) MBC, (d) MBS and (e) MBSC. 
Table 1. Elemental compositions of the samples (KGB, MB, MBC, MBS and MBSC) conducted by EDX analyses.

\begin{tabular}{ccccccccccc}
\hline & \multicolumn{1}{c}{ KGB } & \multicolumn{4}{c}{ MB } & \multicolumn{2}{c}{ MBC } & \multicolumn{2}{c}{ MBS } & \multicolumn{2}{c}{ MBSC } \\
Element & wt.\% & at.\% & wt. $\%$ & at. $\%$ & wt. $\%$ & at. $\%$ & wt. $\%$ & at.\% & wt.\% & at.\% \\
\hline \hline $\mathrm{C}$ & 75.07 & 81.74 & 34.74 & 52.38 & 75.28 & 87.09 & 27.69 & 43.72 & 79.15 & 89.36 \\
$\mathrm{~N}$ & 04.16 & 0.388 & 02.81 & 03.63 & 05.27 & 05.23 & 02.62 & 03.55 & 03.67 & 03.55 \\
$\mathrm{O}$ & 14.81 & 12.10 & 28.11 & 31.82 & 04.25 & 03.70 & 32.20 & 38.18 & 04.26 & 03.61 \\
$\mathrm{Na}$ & 00.00 & 00.00 & 00.57 & 00.45 & 00.00 & 00.00 & 00.68 & 00.56 & 00.06 & 00.03 \\
$\mathrm{Mg}$ & 00.14 & 00.08 & 00.40 & 00.30 & 00.04 & 00.02 & 00.44 & 00.35 & 00.08 & 00.04 \\
$\mathrm{Al}$ & 00.02 & 00.01 & 00.30 & 00.20 & 00.04 & 00.02 & 00.33 & 00.23 & 00.04 & 00.02 \\
$\mathrm{Si}$ & 01.46 & 00.68 & 00.64 & 00.42 & 00.54 & 00.27 & 03.08 & 02.08 & 01.04 & 00.50 \\
$\mathrm{P}$ & 00.07 & 00.03 & 00.17 & 00.10 & 00.01 & 00.01 & 00.14 & 00.09 & 00.03 & 00.01 \\
$\mathrm{~S}$ & 00.53 & 00.21 & 00.38 & 00.21 & 00.12 & 00.05 & 00.27 & 00.16 & 00.08 & 00.03 \\
$\mathrm{Cl}$ & 00.52 & 00.19 & 00.65 & 00.33 & 00.06 & 00.02 & 00.16 & 00.09 & 00.05 & 00.02 \\
$\mathrm{~K}$ & 03.00 & 01.00 & 00.13 & 00.06 & 00.06 & 00.02 & 00.09 & 00.04 & 00.05 & 00.02 \\
$\mathrm{Ca}$ & 00.23 & 00.07 & 00.13 & 00.06 & 00.07 & 00.03 & 00.00 & 00.00 & 00.05 & 00.02 \\
$\mathrm{Fe}$ & 00.00 & 00.00 & 30.98 & 10.05 & 14.24 & 03.54 & 32.31 & 10.97 & 11.45 & 02.78 \\
$\mathrm{Matrix}$ & Correct. & ZAF & Correct. & ZAF & Correct. & ZAF & Correct. & ZAF & Correct. & ZAF \\
\hline
\end{tabular}

The strong band appearing at $1091 \mathrm{~cm}^{-1}$ in all the samples could be assigned to $\mathrm{Si}-\mathrm{O}-\mathrm{Si}$ stretching mode, which was found to become stronger in the samples amended with $\mathrm{SiO}_{2}$ (i.e. MBS and MBSC). Interestingly, high temperature calcination did not affect this band and it remained in all the samples. The band appearing around $3400 \mathrm{~cm}^{-1}$ could correspond to the stretching of $-\mathrm{OH}$ group in water molecule, which is consistent with the studies reported earlier [7, 18]. Thus, the FT-IR study revealed the complexity of all the samples and variations of some constituents during magnetization and $\mathrm{SiO}_{2}$ amendment.

\subsection{SEM images}

Fig. 3 presents the SEM images of the samples (KGB, MB, MBC, MBS and MBSC). The presence of internal pores and cracks (micropores and mesopores) in KGB contributed to its surface area and pore volume, as shown in Fig. 3a. A good dispersion of $\mathrm{Fe}_{3} \mathrm{O}_{4}$ was confirmed in the biochar, as shown in Fig. 3b, which helped to keep the morphological structure of the biochar. Similarly, a recent study [19] revealed the successful dispersion of nanosized $\gamma-\mathrm{Fe}_{2} \mathrm{O}_{3}$ in the biochar matrix. Calcination could cause coarsening of the particles due to thermal treatment process [17]. In this study, calcination further decomposed the sample which appeared to be composed of polyhedral and heterogeneous particles of different sizes, as shown in Fig. 3. Morphological and mineralogical differences were also observed in the samples calcined under different environments [12, 17]. Similarly, both the calcined products (Fig. 3c and Fig. 3e) were observed with variable compositions, as given in Table 1.

Calcination was considered to decrease the surface area of the samples, which tended to sintering and crystallizing [20]. But in this study, the surface areas of the calcined products were dramatically increased as compared to the uncalcined samples, as shown in Table 2. Interestingly, the surface area of MB increased from $45 \mathrm{~m}^{2} \cdot \mathrm{g}^{-1}$ to $336 \mathrm{~m}^{2} \cdot \mathrm{g}^{-1}$ after higher temperature calcination and the carbon content remained the same as in KGB (Table 1). Similarly, the surface area of MBSC increased from $45 \mathrm{~m}^{2} \cdot \mathrm{g}^{-1}$ to $125 \mathrm{~m}^{2} \cdot \mathrm{g}^{-1}$ after calcination, as given in Table 2. The reason could be the decomposition of organics which also affected the magnetic properties of the prepared samples. Thus, calcination caused significant variations in BET surface area, mean pore diameter as well as in pore volume of the samples. 
Table 2. BET surface area, total pore volume and mean pore diameter of the samples.

\begin{tabular}{cccc}
\hline Sample & $\begin{array}{c}\text { SA } \\
{\left[\mathrm{m}^{2} \cdot \mathrm{g}^{-1}\right]}\end{array}$ & $\begin{array}{c}\text { Total pore volume } \\
\mathrm{V}_{\mathrm{p}}\left[\mathrm{cm}^{3} \cdot \mathrm{g}^{-1}\right]\end{array}$ & $\begin{array}{c}\text { Mean pore } \\
\text { diameter }[\mathrm{nm}]\end{array}$ \\
\hline \hline KGB & 35.34 & 0.005 & 6.5 \\
MB & 45.83 & 0.024 & 2.1 \\
MBC & 336.79 & 0.021 & 2.5 \\
MBS & 45.37 & 0.024 & 2.1 \\
MBSC & 125.17 & 0.008 & 2.7 \\
\hline
\end{tabular}

\subsection{Saturation magnetization}

The saturation magnetizations of $\mathrm{MB}, \mathrm{MBC}$, MBS and MBSC samples are presented in Fig. 4. Interestingly, the saturation magnetization of both the composite materials was found to increase after higher temperature calcination. Similarly, in our previous study [12], we found that nitrogen environment calcination increased the saturation magnetization of magnetic honeycomb cinders. Saturation magnetization of MB and MBS increased from $7.8 \mathrm{Am}^{2} \cdot \mathrm{kg}^{-1}$ and $12.8 \mathrm{Am}^{2} \cdot \mathrm{kg}^{-1}$ to $74.6 \mathrm{Am}^{2} \cdot \mathrm{kg}^{-1}$ and $85.6 \mathrm{Am}^{2} \cdot \mathrm{kg}^{-1}$, respectively, which was quite comparable to the pure $\mathrm{Fe}_{3} \mathrm{O}_{4}$ saturation magnetization [21]. The reason of higher saturation magnetization in calcined samples could be the presence of more superparamagnetic constituents. However, the presence of non-magnetic phase (i.e. iron-oxide) in MB and MBS could cause the lower saturation magnetization behavior. Materials with superparamagnetic behavior can be easily collected by external magnetic field and have wide application potential in environmental remediation measures [7, 12, 17].

\subsection{TGA-DSC investigation}

Fig. 5 (a to c) shows the TGA and DSC results of MB, MBC, MBS and MBSC samples. Generally, there are three stages of thermal degradation of samples, as described in details [7, 19, 22]. The TGA analysis (Fig. 5a to Fig. 5c) of both calcined and uncalcined samples showed a stable weight loss at temperature below $120{ }^{\circ} \mathrm{C}$ and similar to $\mathrm{Fe}_{3} \mathrm{O}_{4} @ \mathrm{SiO}_{2}$-MWCNTs distribution patterns [23]. In this study, comparison of the TGA curves showed that both the calcined samples

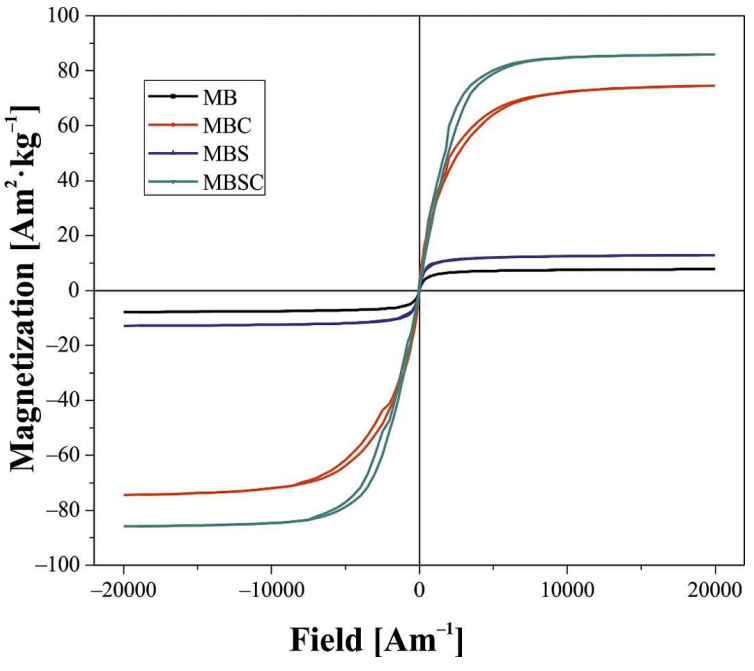

Fig. 4. Saturation magnetization of MB, MBC, MBS and MBSC samples under the applied magnetic field ( -20000 Oe to 20000 Oe).

preserved higher residual mass ( $>95 \%)$ and turned out to be thermally more stable as compared to the uncalcined ones. It revealed that all the samples including calcined and uncalcined were characterized by higher thermal stability, as compared to the other materials studied earlier [24, 25]. DSC values of the samples also displayed the same distribution patterns, which was attributed to the loss of surface and bound water during higher temperature calcination. Thus, TGA-DSC analyses confirmed the presence of higher residual mass in the calcined samples, which were thermally more stable and can potentially be used in environmental remediation measures.

\section{Conclusions}

Selective calcination of magnetic biochar amended with $\mathrm{SiO}_{2}$ demonstrated significant textural, morphological and mineralogical variations of the material, as revealed in characterization analyses. Elimination of $\mathrm{Fe}_{3} \mathrm{O}_{4}$ and the formation of new phases were more obvious in XRD peaks. In addition, saturation magnetization of the biochar further increased and the calcined samples turned out to be thermally more stable as compared to the magnetic biochar. Therefore, selective calcination could be used for effective preparation 

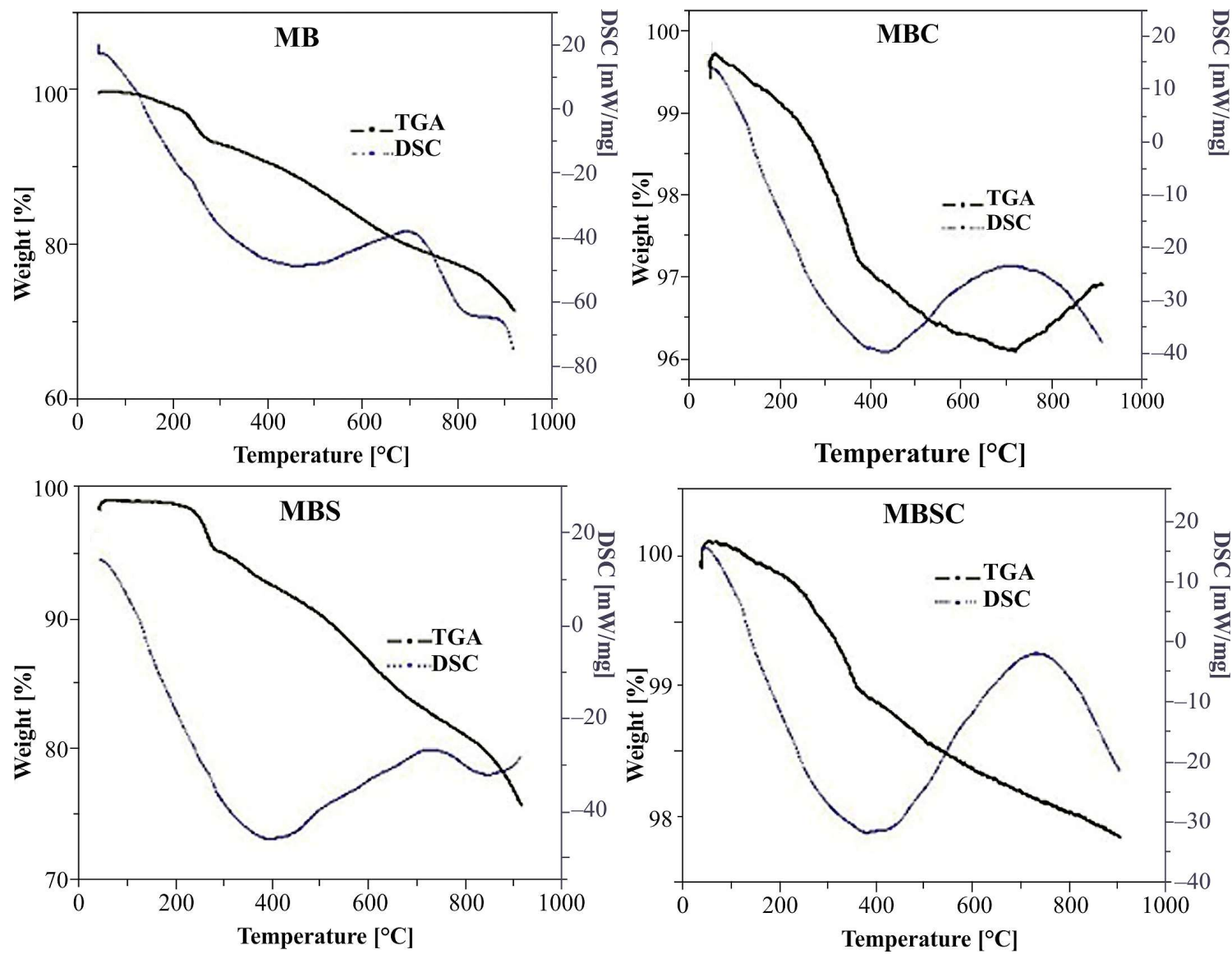

Temperature $\left[{ }^{\circ} \mathrm{C}\right]$

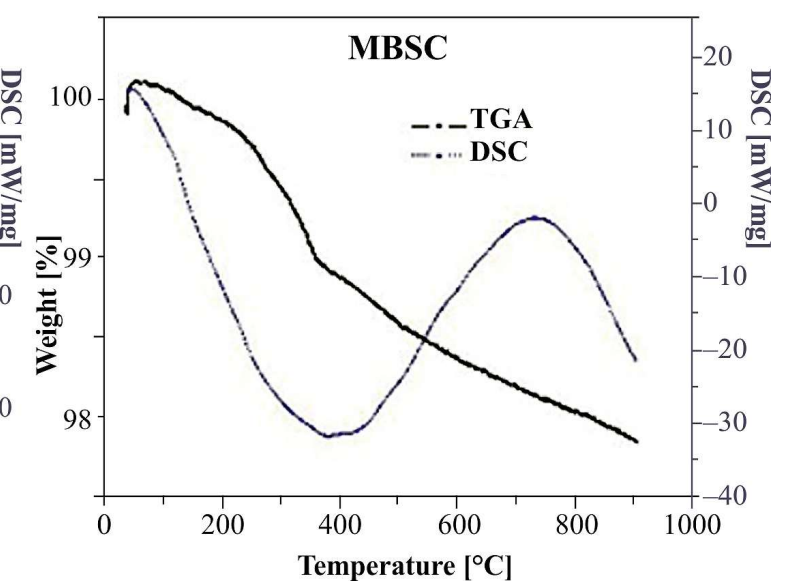

Fig. 5. Thermogravimetric analyses (TGA) of MB, MBC, MBS and MBSC samples.

of the material in environmental remediation measures.

\section{Acknowledgements}

This work is based upon the research supported by the Major Science and Technology Program for Water Pollution Control and Treatment of China (No. 2014ZX07101-012-04) and the National Natural Science Foundation of China (No. 21477108). S.A. Baig also acknowledges the Higher Education Commission (HEC) of Pakistan for the award under "partial support for $\mathrm{PhD}$ studies abroad" and the "start-up research grant".

\section{References}

[1] Johnson M.C., WANG J., Li Z., Lew C.M., YAN Y., Mat. Sci. Eng. A-Struct., 456 (2007), 58.

[2] Diaz-Parralejo A., Macias-Garcia A., Ortiz A.L., Cuerda-Correa E. M., J. Non-Cryst. Solids, 356 (2010), 175.

[3] Mahmood T., Din S., Naeem A., Mustafa S., Waseem M., Hamayun M., Chem. Eng. J., 192 (2012), 90.
[4] Soyer E., Akgiray Ö., Eldem N.Ö., SAatci A.M., CLEAN-Soil Air Water, 41 (2013), 325.

[5] CHEN B., CHEN Z., LV S., Bioresource Technol., 102 (2011), 716.

[6] Chen Y.N., Chai L.Y., Shu Y.D., J. Hazard. Mater, 160 (2008), 168.

[7] Baig S.A., Zhu J., Muhammad N., Sheng T., XU X., Biomass Bioenerg., 71 (2014), 299.

[8] Mohan D., Sarswat A., OK Y.S., Pittman J.C.U., Bioresource Technol., 160 (2014), 191.

[9] BAig S.A., Sheng T., Hu Y., XU J., XU X., CLEANSoil Air Water, 43 (2013), 13.

[10] Tora A., Danaoglu N., Arslan G., CengelOGLU Y., J. Hazard. Mater., 164 (2009), 271.

[11] Luo H., Huang S., Luo L., Wu G., LiU Y., J. Hazard. Mater., 227 - 228 (2012), 265.

[12] Baig S.A., Zhu J., Tan L., Xue X., Sun C., Xu X., Chem. Eng. J., 257 (2014), 1.

[13] Zheng T., Pang J., TAn G., He J., Mcpherson G.L., LU Y., JOHN V.T., ZHAN J., Langmuir, 23 (2007), 5143.

[14] Asaoka S., Hayakawa S., Kim K.-H., TaKeda K., Katayama M., Yamamoto T., J. Colloid Interf. Sci., 377 (2012), 284. 
[15] Tian Y., Wu M., Lin X., Huang P., Huang Y., $J$. Hazard. Mater., 193 (201), 10.

[16] Komolwanich T., TatiJarern P., PrasertWASU S., Khumsupan D., Chaisuwan T., LuenGNARUEmitchai A., WongKasemutt S., Cellulose, 21 (2014), 1327.

[17] Baig S.A., Sheng T., Sun C., Xue X., Tan L., Xu X., Plos One, 9 (2014), e100704.

[18] Sheng T., Baig S.A., Hu Y., Xue X., Xu X., Arab. J. Chem., 7 (2014), 27.

[19] Zhang M., GAO B., VARnoOsfaderon S., HEBARD A., YAO Y., INYANG M., Bioresource Technol., 130 (2012), 457.

[20] Shahar A., Young E.D., Manning C.E., Earth Planet. Sci. Lett., 268 (2008), 330.
[21] Tan L., Xu J., Xue X., Lou Z., Zhu J., Baig S.A., XU X., RSC Adv., 4 (2014), 45920.

[22] Mubarak N.M., Kundu A., Sahu J.N., AbdulLAH E.C., JAYAKUMAR N.S., Biomass Bioenerg., 61 (2014), 265.

[23] Zhou L., Pan S., Chen X., Zhao Y., Zou B., JIN M., Chem. Eng. J., 257 (2014), 10.

[24] Hong P.Z., Li S.D., Ou C.Y., Li C.P., Yang L., Zhang C.H., J. Appl. Polym. Sci., 105 (2007), 547.

[25] Jiang F., Wang X., Wu D., Appl. Energ., 134 (2014), 456.

Received 2015-12-16 Accepted 2016-06-01 